\title{
Plasma and Cerebrospinal Fluid Monoamine Metabolism in Patients with Chronic Fatigue Syndrome: Preliminary Findings
}

\author{
Mark A. Demitrack, Philip W. Gold, Janet K. Dale, \\ Dean D. Krahn, Mitchel A. Kling, and Stephen E. Straus
}

The syndrome of chronic fatigue, feverishness, diffuse pains, and other constitutional complaints, often precipitated by an acute infectious illness and aggravated by physical and emotional stressors, has a lengthy history in the medical literature. The Centers for Disease Control $(C D C)$ recently formulated a case definition, renaming the illness "chronic fatigue syndrome." Nevertheless, there remain few biological data that can validate the existence of this syndrome as distinct from a wide variety of other, largely psychiatric disorders, and little understanding of its pathogenesis. In the present study, basal plasma and cerebrospinal fluid levels of the monoamine metabolites, 3-methoxy-4-hydroxyphenylglycol (MHPG), 5-hydroxyindoleacetic acid (5-HIAA), and homovanillic acid (HVA) were determined in 19 patients meeting $C D C$ research case criteria for chronic fatigue syndrome and in 17 normal individuals. Patients with chronic fatigue syndrome shwwed a significant reduction in basal plasma levels of MHPG and a significant increase in basal plasma levels of 5-HIAA. Although the functional significance of these findings has not been definitively elucidated, they are compatible with the clinical presentation of a syndrome associated with chronic lethargy and fatigue, and with evidence of persistent immune stimulation, and lend support to the idea that chronic fatigue syndrome represents a clinical entity with potential biological specificity.

\section{Introduction}

The syndrome of chronic fatigue, feverishness, diffuse pains, and other constitutional complaints, often precipitated by an acute infectious illness and aggravated by physical and emotional stressors, has a lengthy history (Manningham 1750; Beard 1869). Alternate views have considered this disorder either to represent the physiological aftermath of an acute infectious event or other physical insult in emotionally susceptible individuals, or have emphasized the importance of the infectious onset, with constitutional symptoms

From the Department of Psychiatry, University of Michigan Medical Center, Ann Arbor, Michigan (MAD, DDK); the Clinical Neuroendocrinology Branch, National Institute of Mental Health (PWG, MAK) and the Medical Virology Section, Laboratory of Clinical Investigation, National Institute for Allergy and Infectious Diseases (JKD, SES), National Institutes of Health, Bethesda, MD.

Address reprint requests to Mark A. Demitrack, M.D., Department of Psychiatry, University of Michigan Medical Center, 1500 East Medical Center Drive, Ann Arbor, MI 48109-0116.

Received October 11, 1991; revised July 21, 1992. 
Table 1. Centers for Disease Control (CDC) Case Criteria for Chronic Fatigue Syndrome

Major criteria: (Patient must fulfill major criteria 1 and 2)

1. New onset of persistent or relapsing, debilitating fatigue or easy fatigability that does not resolve with bedrest and is severe enough to impair average daily activity below $50 \%$ of premorbid activity level, lasting for a period of at least 6 months.

2. Exclusion of other clinical conditions or medication effects by appropriate history, physical examination or laboratory tests.

Minor criteria: (Patient must show 6 or more of the symptom criteria and 2 or more of the physical criteria, or 8 or more of the symptom criteria)

Symptom criteria (must have begun at or after onset of the fatigue and must be persistent or recurring)

1. Mild fever or chills

2. Sore throat

3. Painful anterior or posterior cervical or axillary lymph nodes

4. Generalized muscle weakness

5. Myalgias

0. Prolonged postexertional fatigue

7. Headaches

8. Migratory arthralgia

9. Neuropsychological complaints (including photophobia, transient scotomata, forgetfulness, irritability, confusion, depression, poor concentraticn)

10. Sleep disturbance

II. Main symptom complex having an abrupt onset

Physical criteria (must be documented on at least two occasions, at least 1 month apart)

1. Low grade fever (oral $\mathrm{T} 37.6-38.6^{\circ} \mathrm{C}$ or rectal $\mathrm{T} 37.8-38.8^{\circ} \mathrm{C}$ )

2. Nonexudative pharyngitis

3. Palpable anterior or posterior cervical or axillary lymph nodes

(From Holmes et al 1985).

emerging secondary to persistent immune stimulation and/or other lasting pathophysiologic sequelae. Because nu consistent infectious markers have been found in these patients, the hypothesis of a specific infectious etiology has not been universally accepted, nor has it been excluded.

In order to facilitate identification of objective characteristics and improve comparability of research studies, the Centers for Disease Control (CDC) more recently formulated a working case definition, renaming the illness "chronic fatigue syndrome" (Holmes et al 1988). Patients meeting criteria for the syndrome must have persistent or relapsing, debilitating fatigue for at least 6 months in the absence of any medical diagnosis that would explain the clinical presentation. Several symptom criteria are also required for diagnosis (Table 1).

We recently proposed that the substantial clinical overlap between chronic fatigue syndrome and a variety of primary psychiatric illnesses reflects a disturbance in a final common biological pathway that may be precipitated by a variety of pathophysiological antecedents (infectious or noninfectious). We showed that patients with chronic fatigue syndrome demonstrate significant hypocortisolism that seemed likely to reflect a deficiency of the principal hypothalamic stimulus to the pituitary-adrenal axis, namely, corticotropinreleasing hormone (Demitrack et al 1991). These findings were of interest because hypocortisolism itself could contribute to many of the symptoms of chronic fatigue syndrome (e.g., lethargy, fatigue, sleep disturbance, myalgias, arthralgias, enhanced immune activity). Moreover, corticotropin-releasing hormone serves not only as the principal stim- 
ulus to the pituitary-adrenal axis, but is itself a behaviorally active neurohormone whose central administration to animals and nonhuman primates induces signs of physiological and behavioral arousal, including activation of the sympathetic nervous system (Brown et al 1982), hyperresponsiveness to sensory stimuli (Swerdlow et al 1986), and increased locomotion (Sutton et al 1982). Hence, a deficiency of hypothalamic corticotropin-releasing hormone function or release could contribute to the profound lethargy and fatigue that are inherent characteristics of chronic fatigue syndrome, either through direct effects upon the central nervous system or indirectly by causing glucocorticoid deficiency.

We report here a preliminary investigation of basal cerebrospinal fluid and plasma measures of the monoamine metabolites, 3-methoxy-4-hydroxyphenylglycol (MHPG), 5-hydroxyindoleacetic acid (5-HIAA), and homovanillic acid (HVA) in patients with chronic fatigue syndrome and in normal individuals. Several observations originally prompted our interest in examining these neurochemicals. First, from a clinical perspec. tive, it has been suggested that chronic fatigue syndrome may be a form of major depression, an illness in which disturbances in these neurochemicals have been extensively studied. Second, activation of hypothalamic corticotropin-releasing hormone appears to be anatomically and functionally linked to another stress-responsive and arousal-produsing neurotransmitter system, namely, the locus ceruleus-noradrenergic system. Intracerebroventricular administration of corticotropin-releasing hormone increases the spontaneous discharge of locus ceruleus neurons (Valentino et al 1983). Nitroprusside and hemorrhage, two stimuli known to activate hypothalamic corticotropin-releasing hormone neurons, lead to similar degrees of activation of locus ceruleus neurons (Valentino 1988). Furthermore, the $\beta$-adrenoceptor antagonist propranolol inhibits the enhanced conditioned fear response produced by the central administration of corticotropin-releasing hormone (Cole and Koob 1988). Hence, a putative deficiency of hypothalamic corticotropinreleasing hormone may be reflected as a decrease in noradrenergic function. Our interest in the metabolite 5-HIAA is due not only to the established role for its parent neurotransmitter, serotonin, in the mediation of mood, sleep, pain perception, and appetite, but also because, in the periphery, serotonin itself acts as a vasoactive amine, released at sites of inflammation by a variety of stimuli. Multifocal pain and signs of inflammation are common, defining features of chronic fatigue syndrome. $\mathbf{A}$ final reason for studying monoamine neurotransmission stems from the emerging understanding of the bidirectional communication occurring between the nervous and the immune systems (Dunn 1988). Although a wide array of compounds participate in this communication process, certain observations suggest a significant role for the monoamines. For instance, perturbations of the immune system in the periphery may be associated with significant alterations in central monoaminergic activity (Bhattacharya et al 1988). Furthermore, peripheral immunocytes possess receptors for a variety of monoamine neurotransmitters, and other lymphoid tissues are extensively innervated by automatic neurons (Livnat et al 1985).Assessing the integrity of monoaminergic neurotransmission in the central nervous system and in the periphery may, therefore, help extend and clarify the results of our initial studies, and orient future work in this area.

\section{Subjects}

Only patients who had met the CDC research case definition for the chronic fatigue syndrome, and in whom no other medical diagnosis could be established, were included in the present study. Selection was also based on availability for the study duration, 
ability to comply with the strict dietary and medication-free constraints of the study, and willingness to participate. Except îor the severity criteria in the established case definition, illness severity per se was not considered in patient selection. Formally diagnosable current or past psychiatric illnesses are present in well over half of all reported case series of chronic fatigue syndrome. At the present time, however, a descriptive algorithm for the differentiation of primary psychiatric illnesses from chronic fatigue syndrome remains elusive in many, if not all, cases. Hence, it is difficult and often impossible to determine whether a psychiatric disorder is fully explanatory of the clinical presentation, a secondary manifestation of a primary disease process, or a coincident disease that modulates the presentation and course of the fatigue state. In our patient recruitment, rather than attempt to establish a definitive distinction between psychiatric illness and CFS per se, we chose as a systematic methode zy an approach that characterizes psychopathology a priori, and therefore allows, a posteriori, an examination of the interaction of psychopathology with the definition of chronic fatigue syndrome.

Using the above selection criteria, 19 patients were selected in a random fashion from among a larger cohort of 127 patients recruited between 1979 and 1988 at the National Institutes of Health (NIH) and participating in ongoing evaluations of chronic fatigue. The demographic and historical features of the study population were comparable with and representative of prior reported populations of chronic fatigue syndrome patients from this and other centers (Tobi et al 1982; Jones et al 1985; Straus et al 1985, 1988a). Of the 19 patients, 13 were women. The mean age for the entire group was $36.4 \pm 1.9$ years, and the mean duration of illness was $7.4 \pm 1.1$ years (range 1.8-19.9 years). At the time of entry into this study, 6 were employed full or part time and 13 were unemployed. All patients were fully ambulatory and not confined to bed. In 18 of the 19 patients, the syndrome was precipitated by an acute febrile illness. In 7 subjects, the presence of acute infectious mononucleosis was documented ty appropriate clinical and laboratory evaluation. In the remaining 11 , the illness was documented by either clinical or laboratory report of the patient's referring physician, though a specific pathogen was not always indicated. All patients had undergone serial physical examinations and laboratory evaluations, described elsewhere (Demitrack et al 1991), for at least 2 years prior to study, during which time no alternative medical diagnoses could be established. Fifteen of the 19 patients had previously undergone epicutaneous skin testing with an aeroallergen/food battery screen (Straus et al 1988a) before entry into the present study.

Seventeen normal volunteers (eight women, nine men) were recruited by newspaper advertisement from the Washington D.C. area. The mean group of the normal volunteers was $31.2 \pm 2.2$ years. Patient and normal volunteer study groups were matched for age within one decade (two-tailed, unpaired $t$-test, NS) and did not differ significantly in their gender distribution (Fisher's exact test, NS). All normal volunteers provided detailed medical histories and underwent physical and laboratory examinations, which revealed no evidence of current or past neurological, endocrinological, cardiovascular, hepatic, renal, or hematological disease. An unstructured psychiatric interview revealed no evidence of current or past history of psychiatric illness or history of chronic fatigue in the volunteers themselves or in their first-degree relatives.

All subjects abstained from the use of medications, alcohol, tobacco, and caffeine for at least 2 weeks prior to the study and were maintained on a modified low monoamine diet for at least 3 days prior to the study. Female subjects were studied during the follicular phase of the menstrual cycle (days 1 through 10). All testing was performed on an inpatient basis at the NIH Clinical Center. The study protocol was reviewed and approved by NIH 
Institutional Review Boards. All subjects gave written informed consent prior to participation in the study. All of the patients and normal volunteers had previously been participants in our studies of hypothalamic-pituitary-adrenal function in patients with chronic fatigue syndrome (Demitrack et al 1991).

\section{Methods}

All subjects were admitted to the NIH Clinical Center on the evening prior to the study, and were maintained on strict bedrest for at least 6 continuous hr prior to the study. Only water was consumed after midnight. An indwelling intravenous catheter was inserted into an antecubital vein at 8:00 AM. After $1 \mathrm{hr}$, and immediately prior to lumbar puncture, a venous blood sample was withdrawn in a prechilled glass tube containing potassium ethylenediaminetetraacetate (EDTA). The blood sample was centrifuged at $+4^{\circ} \mathrm{C}$ within $1 \mathrm{hr}$ of collection and the plasma was decanted and frozen at $-70^{\circ} \mathrm{C}$ until assayed. Lumbar punctures were performed in the lateral decubitus position between 9:00 AM and 9:30 AM. Thirty milliliters of cerebrospinal fluid were withdrawn over 15-30 min. The first $12 \mathrm{ml}$ were collected in a single pool in a chilled polyethylene tube. This pooled fraction was gently mixed, divided into $1 \mathrm{ml}$ aliquots, immediately frozen on dry ice, and then stored at $-70^{\circ} \mathrm{C}$ until assayed.

The norepinephrine metabolite MHPG, the serotonin metabolite 5-HIAA, and the dopamine metabolite HVA were measured by means of high-pressure liquid chromatography with electrochemical detection, as previously described (Seppala et al 1984). Intraassay and interassay variabilities ranged from $5.6 \%$ to $14.7 \%$ and from $4.6 \%$ to $5.8 \%$, respectively.

Prior to lumbar puncture, all patients were interviewed using the Diagnostic Interview Schedule, version IIl-A (DIS-III-A) (Robins and Helzer 1985). To assess the possible misdiagnosis caused by similarity in symptoms between the chronic fatigue syndrome and psychiatric disorders, wis employed a previously described method of scoring the DIS-III-A which was used in classifying psychiatric diagnoses in patients with chronic disease (Lustman et al 1986). Subsequently, the interviews were re-scored, including as behaviorally relevant the symptoms that were explicitly attributed to the fatigue syndrome. Interviews were scored by both methods to yield DSM-III-R diagnoses. When the age of onset of the chronic fatigue syndrome and age at which psychiatric problems occurred were within 1 year of each other, they were considered concurrent.

All data are expressed as mean \pm 1 SEM. Between-group comparisons were performed using two-tailed, unpaired $t$-tests, employing a Bonferroni-adjusted $\alpha$ level to determine statistical significance. Data analyses were performed on a Macintosh SE/30 microcomputer using the Statview 512 + statistical software package and on an Amdahl 470 V/8 mainframe system using the BMDP statistical package.

\section{Results}

\section{Basal Plasma and Cerebrospinal Fluid Measures of Monoamine Metabolites}

Basal plasma levels of MHPG were significantly reduced in patients with chronic fatigue syndrome compared with normals $(8.3 \pm 0.4$ versus $10.8 \pm 0.6 \mathrm{pmol} / \mathrm{mI}$, df $=34$, $t=3.384, p=0.0018$ ), whereas plasma levels of 5-HIAA were significantly elevated $(67.3 \pm 4.2$ versus $37.3 \pm 5.9 \mathrm{pmol} / \mathrm{ml}, \mathrm{df}=34, t=-4.201, p=0.002)$ (Table 
Table 2. Plasma and Cerebrospinal Fluid Monoamine Metabolites in Patients with Chronic Fatigue Syndrome and in Healthy Controls

\begin{tabular}{lllcr}
\hline & & $n$ & Mean & SEM \\
\hline Cerebrospinal fluid & & & & \\
MHPG & Patients & 19 & 41.5 & 1.3 \\
& Normals & 17 & 44.7 & 1.9 \\
5-HIAA & Patients & 19 & 111.6 & 8.1 \\
& Normals & 17 & 95.1 & 10.1 \\
HVA & Patients & 19 & 188.8 & 18.1 \\
& Normals & 17 & 191.6 & 27.7 \\
Plasma & & & & \\
MHPG & Patients & 19 & $8.3^{a}$ & 0.4 \\
& Normals & 17 & 10.8 & 0.6 \\
5-HIAA & Patients & 19 & $67.3^{\circ}$ & 4.2 \\
& Normals & 17 & 37.3 & 5.9 \\
HVA & Patients & 19 & 49.8 & 2.6 \\
& Normals & 17 & 55.7 & 5.6 \\
\hline
\end{tabular}

All sample values are expressed as $\mathrm{pmol} / \mathrm{ml}$.

${ }^{u} p=0.0018 ;{ }^{b} p=0.002 ;$ two-tailed, unpaired t-test; (Bonferroni-adjusted significance level $=0.0083$ ).

2). Interestingly, those patients $(n=10)$ with previous evidence of a positive immediate hypersensitivity response to an epicutaneous aeroallergen/fnnd battery screen tended to have higher levels of plasma 5-HIAA compared with those with no such response (72.0 \pm 5.2 versus $53.6 \pm 9.3 \mathrm{pmol} / \mathrm{ml}, p=0.08$, unpaired, two-tailed $t$-test).

Plasma levels of HVA were no different in the patients with chronic fatigue syndrome compared with normals, nor were there any differences in cerebrospinal fluid monoamine metabolite levels between the patients and normals (Table 2). There were no significant gender-related differences in either the plasma or cerebrospinal fluid monoamine measures (data not shown).

\section{Relation between Psychiatric Diagnosis and Monoamine Measures}

As noted above, because the identification of psychiatric illnesses by standard diagnostic criteria includes many symptoms that are an inherent part of the definition of chronic fatigue syndrome itself (e.g., fatigue, lethargy, sleep disturbance), we first evaluated the structured interview results using restrictive criteria that excluded symptoms attributable to the chronic fatigue syndrome itself. By this method, 5 of 19 patients reported a lifetime history of major depression, 4 a lifetime history of anxiety disorder, and only 1 met criteria for somatization disorder. However, using criteria that included all symptoms as behaviorally relevant, 10 of 19 patients reported a lifetime history of major depressive illness, 4 reported a lifetime history of anxiety disorder, and 3 met criteria for somatization disorder. Given the occurrence of more than one diagnosis in 7 patients, a total of 13 of the 19 met criteria for a lifetime history of psychiatric illness. In 7 of the subjects, psychiatric illness appeared to precede the onset of the chronic fatigue syndrome. These findings are similar to those reported previously by members of our group and most others in studies of patients with chronic fatigue (Taerk et al 1987; Manu et al 1988, 1989; Kruesi et al 1989; Gold et al 1990; Hickie et al 1990). Although the comparison groups 
were small, there were no evident trends nor statistically significant relationships between a lifetime history of psychiatric illness (using either the restrictive or the inclusive criteria) or its onset relative to the onset of the chronic fatigue syndrome and any of the biological indices studied (two-tailed, unpaired $t$-test comparisons, data not shown).

\section{Discussion}

Patients with chronic fatigue syndrome demonstrated a significant reduction in mean basal plasma levels of the norepinephrine metabolite, MHPG, and a significant elevation in mean basal plasma levels of the serotonin metabolite, 5-HIAA, when compared with normal individuals. Corresponding levels of these metabolites in the cerebrospinal fluid of patients with chronic fatigue syndrome were normal.

These results contrast with the majority of studies of monoamine function in patients with melancholic depression, in whom elevated plasma norepinephrine and MHPG (Wyatt et al 1971; Esler ot al 1982; Lake et al 1982; Jimerson et al 1983; Barnes et al 1983), increased levels of cerebrospinal fluid and urinary MHPG (Koslow et al 1983), and reduced levels of cerebrospinal fluid 5-HIAA (van Praag et al 1970) have generally been detected. Furthermore, we observed no relationship between the presence or absence of a history of formally diagnosed psychiatric illness and the disturbances in monoamine metabolism in patients with chronic fatigue syndrome. Although the mean length of illness in the patients studied here was slightly longer than 7 years, there was no relation between overall duration of illness and any of the biological measures we report. These findings, however, may not generalize to patients with a shorter course of illness. A female preponderance of chronic fatigue syndrome has been reported in the literature. Despite the presence of a nonsignificantly larger percentage of female patients compared with female normals in our sample populations, we were unable to perceive a gender-related effect on the findings we report here. It should be cautioned, however, that the small sample sizes make a firm conclusion on the role of gender as a confounding variable tentative at this time. Though the causes and implications of these findings are not completely clear, a number of issues deserve comment and may help to sugb - st directions for future study.

Norepinephrine is the principal neurotransmitter released from nerve endings of the sympathetic nervous system. Plasma levels of norepinephrine and its metabolites are determined by a variety of competing factors including the level of sympathetic nervous system activity and the rates of intraneuronal norepinephrine synthesis and release, the rates of intraneuronal reuptake and diffusion into extraneuronal tissues, and the site and methodology of sampling (Eisenhofer et al 1988). The principal metabolites of norepinephrine include the deaminated compounds, MHPG, and dihydroxyphenylglycol (DHPG). The basal and stimulated levels of plasma norepinephrine and DHPG are believed to reflect the intraneuronal disposition of norepinephrine and hence, may be the most sensitive indices of sympathetic nervous activity (Goldstein et al 1988). The functional significance of plasma MHPG is less clear, as a number of factors are known to influence its basal activity, including a phase shift in its circadian secretion, hemodynamic status, and the level of ongoing physical activity of the individual (Post et al 1984). In detecting reduced MHPG levels, we cannot rule out the presence of a circadian phase disturbance in the patients we studied. On the other hand, no subject was hemodynamically compromised, making this an unlikely source of interpretive error. All subjects, including the healthy 
controls, were on strict bedrest for at least 6 continuous hr prior to lumbar puncture. However, because reduction in overall physical activity is a feature of chronic fatigue syndrome, this factor may contribute to the reduced basal plasma MHPG levels we found.

Whether the reduction in basal plasma MHPG reflects an intrinsic diminution in sympathoneural activity in chronic fatigue syndrome cannot be definitively concluded from this study. However, the present observations take on particular interest when considered in the context of an illness in which there is also evidence of impaired activation of the hypothalamic-adrenal axis, presumably mediated by a reduction in the functional activity of hypothalamic corticotropin-releasing hormone (Demitrack et al 1991). As noted previously, corticotropin-releasing hormone serves not only as the principal stimulus to the pituitary-adrenal axis, but is also a behaviorally active neurohormone whose central administration to animals and nonhuman primates induces signs of physiological and behavioral arousal, including activation of the sympathetic nervous system (Brown et al 1982). These effects of corticotropin-releasing hormone are, in part, exerted through the established anatomical and functional connections with the principal arousal-producing neurotransmitter pathways involving the locus ceruleus-noradrenergic system (Valentino et al 1983; Valentino 1988; Cole and Koob 1988). Together with the results of the current report, these observations suggest that more detailed studies of basal and stimulated activity of the sympathetic nervous system in patients with chronic fatigue syndrome are warranted.

5-HIAA represents the principal degradation product of serotonin metabolism. In humans, approximately $90 \%$ of serotonin is located in the mucosa of the gastrointestinal tract. Much of the remainder is stored in platelets or synthesized in neurons of the central nervous system, in the latter case where it functions as a neurotransmitter. Central nervous system serotonin activity is thought to play an important role in several key physiological processes, including the regulation of sleep, mood, and the conscious perception of pain. However, a disturbance in central serotonin metabolism in patients with chronic fatigue syndrome seems unlikely from the present results, as ccrebrospinal fluid levels of 5HIAA in the patients we describe here were no different from normal.

How might the elevation in plasma levels of 5-HIAA be understood? One of the principal physiological roles for serotonin in the periphery is as a vasoactive amine, released at sites of inflammation by a variety of stimuli. Similar to histamine, serotonin can induce smooth muscle contractility and increase capillary permeability, and is thought to play an important role in the development of both immediate and delayed-type hypersensitivity reactions. For instance, in mice, serotonin stimulates the late-acting T-cells which mediate the effects of delayed-type hypersensitivity (Ameisen et al 1989). Furthermore, release and uptake of serotonin metabolites may be altered in a number of atopic and allergic conditions in humans (Ring and O'Connor 1979; Ring et al 1980; Malmgren et al 1982; Little et al 1983; Ring and Dorsch 1985). Patients with chronic fatigue syndrome are reported to have a higher incidence of allergic conditions (Straus et al 1988b). Indeed, it has been speculated that heightened allergic responsiveness may be a risk factor for the development of the syndrome. In particular, the diverse clinical and immunologic features have been argued to reflect an ongoing state of immune activation, with the persistent release of cytokines and inflammatory mediators in response to antigenic challenge (Cheney et al 1989; Chao et al 1990; Landay et al 1991). The observation, in the present study, of a trend toward higher levels of plasma 5-HIAA in those subjects with a history of immediate hypersensitivity to epicutaneous skin testing is in accord with these ideas. In addition to its pro-inflammatory effects, peripherally 
secreted serotonin also potentiates the nociceptive effects of painful stimuli in both animals and humans (Nakano and Taira 1976; Giordano and Rogers 1989; Jensen et al 1990; Jensen et al 1991). Thus, an increase in peripheral levels of 5-HIAA in patients with chronic fatigue syndrome may reflect an increased turnover of serotonin, responsible in part for both the heightened allergic responsiveness in these patients as well as the symptomatic complaints of widespread musculoskeletal pain which characterize this syndrome.

A variety of immunologic abnormalities have been reported in patients with chronic fatigue syndrome. These abnormalities are diverse in character, relatively modest in magnitude, and of uncertain pathophysiological significance. Of particular note, none of these abnormalities have been shown to bear any relationship to the clinical severity of the syndrome itself. Initial reports described abnormal patterns of antibody responses to a variety of infectious agents and nonspecific elevations in titers of antinuclear antibodies and immune complexes (Tobi et al 1982; Jones et al 1985; Straus et al 1985). Subsequent studies showed patterns of immunoglobulin subclass deficiencies, diminished natural killer cell number and function, alterations in T-cell subset number and function, and abnormalities in serum cytokine levels or in vitro cytokine production (Kibner et al 1985; Caligiuri et al 1987; Komaroff et al 1988; Cheney 1989; Lloyd et al 1989; Straus et al 1989; Chao et al 1990; Klimas et al 1990; Jones 1991). Landay et al (1991) and members of our group (Straus et al, unpublished observations) have recently described abnormalities in lymphocyte surface antigen cell adhesion marker expression suggesting a persistent immune stimulation in patients with chronic fatigue syndrome. The biological mechanisms that mediate these various immune disturbances are not clear. However, it is of interest to the present study that peripheral immunocytes and other lymphoid tissues possess receptors for a variety of monoamine neurotransmitters and receive an extensive innervation from the autonomic nervous system (Livnat et al 1985; Bonnet et al 1987; Fuchs et al 1988; van Tits et al 1990). Interactions between monoaminergic neurotransmitters and components of the immune system are therefore possible at both central and peripheral levels (Livnat et al 1985). This may occur through either central modulation of autonomic outflow to lymphoid tissues, or via changes in receptor responsiveness on the relevant target tissue. Whether the disturbances in monoamine function seen in the present study account for any of the immune abnormalities noted above, however, remains to be determined.

It has been suggested that chronic fatigue syndrome is frequently precipitated by an acute infection. It is, therefore, relevant to speculate whether infections could alter neurotransmitter levels, und whether such alterations, in turn, could influence neuroendocrine or neuroimmune function. Southern and Oldstone (1986) have conducted a series of studies that bear on these speculations and which have led to the concept that chronic infection can disturb organismic homeostasis without damaging the morphologic appearance or the essential biological machinery of cells. Using a poorly cytocidal murine RNA virus. lymphocytic choriomeningitis virus, they showed that persistent infection alters levels of acetylcholine synthesis in neuroblastoma cells in vitro (Oldstone et al 1977). In vivo, this virus displays a specific tropism for anterior pituitary somatotrophs, leading to a syndrome of retarded growth and abnormal glucose regulation in mice, due to a specific impairment in the secretion of growth hormone (Oldstone et al 1982). Moreover, persistent infection with this and several other agents, including the human immunodeficiency virus, is known to induce a variety of derangements of immune function. It should be noted that in the present study, all but one patient reported an acute 
infectious precipitant to their chronic fatigue. Hence, whether idiopathic chronic fatigue states presenting in the aftermath of an acute infection display psychobiological characteristics that differ from those fatigue states developing in the absence of an identifiable infectious stressor cannot be determined from the present data.

For many years, it had been proposed that the protracted debility occurring in the aftermath of an acute infection reflects the presence of preexisting psychological morbidity (Imboden et al 1959). Indeed, this argument can be made for chronic fatigue syndrome as well. Most studies discerned that the prevalence of formally diagnosed psychiatric illness was higher in patients with either chronic fatigue or chronic fatigue syndrome than in the general population, and often preceded the onset of the fatigue syndrome (Taerk et al 1987; Manu et al 1988, 1989; Kruesi et al 1989; Gold et al 1990). In only one study was the prevalence of psychiatric illness reported to be comparable to that of the general population, and to follow, rather than precede the development of the chronic fatigue (Hickie et al 1990). However, in the absence of reliable biological measures to identify this syndrome, and given that most established psychiatric diagnostic systems include fatigue as a symptom of psychiatric disorder, the use of phenomenological criteria alone has introduced an unavoidable circularity of reasoning in establishing a definitive distinction between chronic fatigue that is separate from, as opposed to a feature of, a psychiatric illness. Though results of the present investigation do not validate the construct of chronic fatigue syndrome as a discrete medical condition, they suggest specific biological differences between patients with this illness and the majority of patients with the melancholic form of major depression.

We conclude that, given the growing body of data that implicate alterations in central and peripheral neurotransmitter function in the pathophysiology of behavioral disorders, the strict etiologic distinction between either "psychological" or "physical" causes in our understanding of this puzzling syndrome no longer seems entirely useful.

\section{References}

Ameisen J-C, Meade R, Askenase PW (1989): A new interpretation of the involvement of serotonin in delayed-type hypersensitivity. $J$ Immunol 142(9):3171-3179.

Barnes RF, Veith RC, Borson S, Verhey J, Raskind MA, Halter JB (1983): High levels of plasma catecholamines in dexamethasone-resistant depressed patients. Am J Psychiatry 140(12):16231625.

Beard G (1969): Neurasthenia, or nervous exhaustion. Boston Med Su $g$ J III(13):217-221.

Bhattacharya SK, Das N, Rao PJRM (1988); Brain monoamines during carrageenan-induced acute paw inflammation in rats. J Pharm Pharmacol 40:518-520.

Bonnet M, Lespinats G, Burtin C (1987): Evidence for serotonin (5HT) binding sites on murine lymphocytes. Int J Immunopharmacol 9(5):551-558.

Brown MR, Fisher LA, Spiess J, Rivier C, Rivier J, Vale W (1982): Corticotropin-releasing factor: Actions on the sympathetic nervous system and metabolism. Endocrinology 111:928-931.

Caligiuri M, Murray C, Buchwald D et al (1987): Phenotypic and functional deficiency of natural killer cells in patients with chronic fatigue syndrome. J Immunol 139:3306-3313.

Chao CC, Gallagher M. Phair J, Peterson PK (1990): Serum neopterin and interleukin-6 levels in chronic fatigue syndrome. $J$ Infect Dis 162:1412-1413.

Cheney PR, Dorman SE, Bell DS (1989): Interleukin-2 and the chronic fatigue syndrome. Ann In Med 110:321.

Cole BJ, Koob GF (1988): Propranolol antagonizes the enhanced conditioned fear produced by CRF. (Abstract) J Cell Biochem (Suppl) 12D:310. 
Demitrack MA, Dale JK, Straus SE, et al (1991). Impaired activation of the hypothalamic-pituitaryadrenal axis in patients with chronic fatigue syndrome. J Clin Eridocrinol Metab (in press).

Dunn AJ (1988): Nervous system-immune system interactions: An overview. J Recept Res 8:(14):598-607.

Eisenhofer G, Goldstein DS, Ropchak TG, Nguyen HQ, Keiser HR, Kopin IJ (1988): Source and physiological significance of plasma 3,4-dihydroxyphenylglycol and 3-methoxy-4-hydroxyphenylglycol. J Autonom Nerv Syst 24:1-14.

Esler M, Turbott J, Schwarz R, et al (1982): The peripheral kinetics of norepinephrine in depressive illness. Arch Gen Psychiatry 39:295-300.

Fuchs BA, Campbell KS, Munson AE (1988): Norepinephrine and serotonin content of the murine spleen: Its relationship to lymphocyte beta-adrenergic receptor density and the humoral response in vivo and in vitro. Cell Immunol 117(2):339-351.

Giordano J, Rogers LV (1989): Peripherally administered serotonin 5-HT3 receptor antagonists reduce inflammatory pain in rats. Eur J Pharmacol 170(1-2):83-86.

Gold D, Bowden R, Sixbey J, et al (1990): Chronic fatigue: A prospective clinical and virologic study. JAMA 264(1):48-53.

Goldstein DS, Eisenhofer G, Stull R, Folio CJ, Keiser HR, Kopin IJ (1988):PLasma dihydroxyphenylglycol and the intraneuronal disposition of norepinephrine in humans. $J$ Clin Invest 81:213-220.

Hickie I, Lloyd A, Wakefield D, Parker G (1990): The psychiatric status of patients with chronic fatigue. Br J Psychiatry 156:534-540.

Holmes GP, Kaplan JE, Gantz NMi, et al (1988): Chronic fatigue syndrome: A working case definition. Ann Int Med 108:387-389.

Imboden JB, Canter A, Cluff LE, Trever RW (1959): Brucellosis III. Psychological aspects of delayed convalescence. Arch Int Med 103:78-86.

Jensen K, Tuxen C, Pedersen-Bjergaard U, Jansen I, Edvinsson L, Olesen J (1990): Pain, wheal and flare in human forearm skin induced by bradykinin and 5-hydroxytryptamine. Peptides 11(6):1133-1138.

Jensen K, Tuxen C, Pedersen-Bjergaard U, Jansen I (1991): Pain, tenderness, wheal and flare induced by substance-P, bradykinin and 5-hydroxytryptamine in humans. Cephalalgia 11(4):175182.

Jimerson DC, Insel TR, Reus VI, Kopin IJ (1983): Increased plasma MHPG in dexamethasoneresistant depressed patients. Arch Gen Psychiatry 40:173-176.

Jones JF (1991): Serologic and immunologic responses in chronic fatigue syndrome with emphasis on the Epstein-Barr virus. Rev Inf Dis 13(Suppl):S26-S31.

Jones JF', Ray G, Minnich LL, Hicks MJ, Kibler R, Lucas DO (1985): Evidence for active EpsteinBarr virus infection in patients with persistent, unexplained illnesses: Elevated anti-early antigen antibodies. Ann Int Med 102(1):1-7.

Kibler R, Lucas D, Hicks MJ, Poulos BT, Jones JF (1985): Immune function in chronic active Epstein-Barr virus infection. J Clin Immunol 5:46-54.

Klimas NG, Salvato FR, Morgan R, Fletcher MA (1990): Immunologic abnormalities in chronic fatigue syndrome. J Clin Microbiol 28:1403-1410.

Komaroff AL, Geiger AM, Wormsley S (1988): IgG subclass deficiencies in chronic fatigue syndrome. Lancet (Jun 4):1288-1289.

Koslow JH, Maas JW, Bowden CL, Davis JM, Hanin I, Javaid J (1983): CSF and urinary biogenic amines and metabolites in depression and mania: A controlled, univariate analysis. Arch Gen Psychiatry 40:999-1010.

Kruesi MJP, Dale JK, Straus SE (1989): Psychiatric diagnoses in patients with the chronic fatigue syndrome. J Clin Psychiatry 50:53-56.

Lake CR, Pickar D, Ziegler MG, Lipper S, Slater S, Murphy DL (1982): High plasma norepinephrine levels in patients with major affective disorder. Am J Psychiatryl 139:1315-1318. 
Landay AL, Jessop C, Lennette ET, Levy JA (1991): Chronic fatigue syndrome: Clinical condition associated with immune activation. Lancet 338(8769):707-712.

Little CH, Stewart AG, Fennessy MR (1983): Platelet serotonin release in rheumatoid arthritis: A study in food-intolerant patients. Lancet 2(8345):297-299.

Livnat S, Felten SY, Carlson SL, Bellinger DL, Felten DL (1985): Involvement of peripheral and central catecholamine systems in neural-immune interactions. $J$ Neuroimmunol 10:5-30.

Lloyd AR, Wakefield D, Boughton CR, Dwyer JM (1989): immunological abnormalities in the chronic fatigue syndrome. Med J Australia 151:122-124.

Lustman PJ, Harper GW, Griffith LS, Clouse RE (1986): Use of the Diagnostic Interview Schedule in patients with diabetes mellitus. $J$ Nerv Ment Dis 174(12):743-746.

Malmgren R, Grubbstrom J, Olsson P, Theorell H, Tomling G, Unge G (1982): Defective serotonin (5-HT) transport mechanism in platelets from patients with endogenous and allergic asthma. Allergy 37(1):29-39.

Manningham R (1750): The Symptoms, Nature, Causes, and Cure of the Febricula, or Little Fever. London: J Robinson.

Manu F, Lane TJ, Matthews DA (1988): The frequency of the chronic fatigue syndrome in patients witn symptoms of persistent fatigue. Akin Int Med 109:554-556.

Maru P, Matthews DA, Lane TJ, et al (1989): Depression among patients with a chief complaint of chronic fatigue. $J$ Affective Disord 17:165-172.

Nakano T, Taira N (1976): 5-Hydroxytryptamine as a sensitizer of somatic nociceptors for painproducing substances. Eur J Pharmacol 38(1):23-29.

Oldstone MBA, Holmstoen J, Welsh RM Jr (1977): Alterations of acetylcholine enzymes in neuroblastoma cells persistently infected with lymphocytic choriomeningitis virus.J Cell Physiol 91:459-472.

Oldstone MBA, Sinha YN, Blount P, et al (1982): Virus-induced alterations in homeostasis: Alterations in differentiated functions of infected cells in vivo. Science 218:1125-1127.

Post RM, Jimerson DC, Ballenger JC, Lake CR, Uhde TW, Goodwin FK (1984): Cerebrospinal fluid norepinephrine and its metabolites in manic-depressive illness. In Post RM, Ballenger JC (eds), Neurobiology of Mood Disorders, Frontiers of Clinical Neuroscience, Vol 1. Baltimore: Williams and Wilkins, pp 539-553.

Ring J, O'Connor $\mathbf{R}$ (1979): In vitro histamine and serotonin release studies in atopic dermatitis. Int Arch Allergy Appl Immunol 58(3):322-330.

Ring J, Dorsch W (1985): Altered releasability of vasoactive mediator secreting cells in atopic eczema. Acta Derm Venereol (Stockh) (Suppl) 114:9-23.

Ring J, Allen DH, Mathison DA, Spiegelberg HL (1980): In vitro releasability of histamine and serotonin: Studies of atopic patients. J Clin Lab Immunol 3(2):85-91.

Robins LN, Helzer JE (1985): The NIMH Diagnostic Interview Schedule, Version III-A. St. Louis: Department of Psychiatry, Washington University School of Medicine.

Seppala T, Scheinin M, Capone A, Linnoila M (1984): Liquid chromatographic assay for CSF catecholamines using electrochemical detection. Acix Pharmacol Toxicol 55:81-97.

Southern P. Oldstone MBA (1986): Medical consequences of persistent viral infection. $N$ Engl J Med 314(6):359-367.

Straus SE, Tosato G, Armstrong G, et al (1985): Persisting illness and fatigue in adults with evidence of Epstein-Barr virus infection. Ann Int Med 102(1):7-16.

Straus SE, Dale JK, Tobi M, et al (1988a): Acyclovir treatment of the chronic fatigue syndrome: Lack of efficacy in a placebo-controlled trial. N Engl J Med 319(26):1692-1698.

Straus SE, Dale JK, Wright R, Metcalfe DD (1988b): Allergy and the chronic fatigue syndrome. J Allergy Clin Immunol 81(5, Pt 1):791-795.

Straus SE, Dale JK, Peter JB, Dinarello CA (1989): Circulating lymphokine levels in chronic fatigue syndrome. $J$ Infect Dis 160:1085-1086. 
Sutton RE, Koob GF, LeMoal M, Rivier J, Vale W (1982): Corticotropin-releasing factol produces behavioural activation in rats. Nature 297:331-333.

Swerdlow NR, Geyer MA, Vale WW, Koob GF (1986): Corticotropin-releasing factor potentiates acoustic startle in rats: Blockade by chlordiazepoxide. Psychopharmacology (Berlin) 1986; 88:147-152.

Taerk GS, Toner BB, Salit IE, Garfinkel PE, Ozersky S (1987): Depression in patients with neuromyasthenia (benign myalgic encephalomyelitis)). Int J Psychiatry Med 17(1):49-56.

Tobi M, Ravid Z, Feldman-Weiss V, et al (1982): Prolonged atypical illness associated with serological evidence of persistent Epstein-Barr virus infection. Lancet i:61-64.

Valentino RJ (1988): Corticotropin-releasing factor (CRF) and physiological stressors alter activity of rat noradrenergic locus coeruleus (LC) neurons in a similar manner. (Abstract) J Cell Bicohem (Suppl) 12D:317.

Valentino RJ, Foote SL, Aston-Jones G (1983): Corticotropin-releasing hormone activates noradrenergic neurons of the locus coeruleus. Brain Res 270(2):363-367.

van Praag H, Korf J, Puite J (1970): 5-Hydroxyindoleacetic acid levels in the cerebrospinal fluid of depressive patients treated with probenicid. Nature 225:1259-1260.

van Tits LJ, Michel MC, Grosse-Wilde H, et al (1990): Catecholamines increase lymphocyte beta2 adrenergic receptors via a beta-2 adrenergic, spleen-dependent process. Am J Physiol 258(1 Pt 1):E191-E202.

Wyatt RJ, Portnoy B, Kupfer DJ, Snyder F, Engleman K (1971): Resing plasma catecholamine concentrations in patients with depression and anxiety. Arch Gen Psychiatry 24:65-70. 